\title{
Biochemistry, Medicinal Properties \& Toxicity of Acacia Nilotica Fruits
}

Kamal Omer Abdalla PhD

Department of Biochemistry \& Molecular Cell Biology, Faculty of Medicine \& Health Sciences, University of Gadarif, P. O. Box 449, Gadarif 32211, Sudan.

*Corresponding Authors: Kamal Omer Abdalla, Department of Biochemistry \& Molecular Cell Biology, Faculty of Medicine \& Health Sciences, University of Gadarif, P. O. Box 449, Gadarif 32211, Sudan.

Received date: December 26, 2020; Accepted date: February 04, 2021; Published date: February 08,2021

Citation: Kamal Omer Abdalla. (2021) Etiology and Clinical Features of Acute Flaccid Paralysis among Children in Gadarif, Sudan. Biomedical Research and Clinical Reviews. 3(2); DOI: 10.31579/2692-9406/040

Copyright:@2021 Kamal Omer Abdalla, This is an open-access article distributed under the terms of the Creative Commons Attribution License, which permits unrestricted use, distribution, and reproduction in any medium, provided the original author and source are credited.

\begin{abstract}
Acacia nilotica tree is native to Africa, the Middle East and the Indian subcontinent. Due to its wide medicinal properties $\&$ uses this plant attracted the attention of many scientists. Its fruits are rich in more than 16 classes of different bioactive molecules \& elements very beneficial for human health. These molecules \& elements include tannins, saponins, phytosterols, cyclitols, alkaloids, anticoagulant agents, regulatory molecules, amines, mucilage, fibers, gums, proteins, various classes of amino acids, carbohydrates, terpenses, crude fats, oils, fatty acids \& minerals. The fruits are used to treat various human disorders including cancers, heart \& liver diseases, diabetes mellitus, malaria, asthma, arrhythmias, acquired immune-deficiency syndrome, coronavirus disease 2019, spleen disorders, for weight loss, colds, congestion, coughs, diarrhea, dysentery, fever, gallbladder, hemorrhage, hemorrhoids, leucorrhea, ophthalmia, sclerosis, tonsillitis, pharyngitis, skin eruptions smallpox and tuberculosis. Acacia nilotica fruits are very beneficial for human health, safe \& effective to treat various human diseases if it is used in appropriate dose \& period. It has been in use in Sudan \& in other parts of Africa widely \& safely for generations to treat various diseases without any reports of toxicity or adverse effects. Moreover, human body has efficient detoxification mechanisms to process and detoxify toxic substances and toxicity in human occurs only from known high toxic substances, overdose, regular and long period of using of herbs or medicines with known side effects. It is apparent that toxicity of acacia nilotica fruits comes from over dosage \& longer than required duration of uses or regular consumption and the solvent/s as well. However, further works are needed by pharmaceutical industries to authentically formulate various components of the of acacia nilotica fruits to be used as medicines \& supplements to enhance the therapy of several human diseases.
\end{abstract}

Key words: acacia nilotica; medicinal properties; toxicity; covid-19; chemistry

\section{Introduction}

In most countries of Africa including Sudan, health facilities \& services are weak in big cities \& not available in most of the rural areas. The situation is more worsening by poverty, high prices of drugs, the lack of paved roads and appropriate transportation facilities; all these together make the movement of patients from the rural areas to city hospitals in Sudan \& Africa to seek medical help a painful journey. In such situations, it is better for urban \& rural residents in Africa \& Sudan to have at home local natural medicines to be used in the times of emergency. African in general \& Sudan in particular is a reservoir of potent potential natural medicines that are not yet fully studied \& utilized. Therefore, there is an urgent need for better knowledge \& uses of these natural medicines in Sudan \& Africa for the use of human wellbeing.

The invention of new techniques of isolation, structure identification \& functional characterizations of plant compounds has assisted tremendously in the development of the field of phytochemistry. Plant compounds have interest as a source of safer or more valuable substitutes than synthetically created antimicrobial agents. In all parts of the world and more precisely in Africa and other least developed countries of Asia, plants are used in traditional medicine to treat different communicable and non-communicable diseases. It is estimated that more that $80 \%$ of people living in developing countries frequently use traditional practices for their primary health care needs $[1,2,3]$.

Acacia nilotica is a tree belongs to the family of Fabaceae native to Africa, the Middle East and the Indian subcontinent $\&$ it is naturalized widely outside its native lands $[4,5]$. Its name was derived from a Greek word meaning "thorn" while nilotica means along the Nile river [6]. Due to its wide medicinal properties $\&$ uses this plant attracted the attention of many scientists. Biochemistry, medicinal properties \& toxicity endorsed to the fruits of the Acacia nilotica are discussed in details in this manuscript.

\section{Biochemical \& Medicinal Properties of Acacia Nilotica Fruits}

Acacia nilotica fruits are rich in more than 16 classes of different bioactive molecules $\&$ elements very beneficial for human health. These molecules \& elements include tannins, saponins, phytosterols, cyclitols, alkaloids, anticoagulant agents, regulatory molecules, amines, mucilage, fibers, 
gums, proteins, various classes of amino acids, carbohydrates, terpenses, crude fats, oils, fatty acids \& minerals [7, 8, 9, 10, 11, 12].

Tannins in acacia nilotica fruits exist as condensed \& hydrolysable tannins with relative proportion of $50 \%$ [11, 13]. Tannins in the fruits include gallic acid, ellagic acids, m-digallic acid, me-ester-n-digallic acid, m-digallic dimer 3,4,5,7-tetrahydroxy flavan-3-ol, oligomer 3,4,7trihydroxy flavan 3,4-diol, flavonoids, protocatechuic acid \& epicatechol $[7,10,11]$. Tannins are natural polyphenols that categorized into different classes as phenolic acids, flavonoids, lignans, and stilbenes. Hydrolysable tannins react \& hydrolyze in water to give different water-soluble products, such as gallic acid, protocatechuic acid and sugars. Gallotannin (tannic acid) is the best condensed tannins (polyflavonoids). Flavonoids are antioxidants involved in plant immunity \& regulation of plant growth $[14,15]$. Antioxidants are protective against cancers, including colon, prostate, breast, endometrial, lung, and pancreatic tumors. It can protect cells and other structures in the body from harmful reactive oxygen species \& thus helping the body develop resistance against infectious agents and scavenge harmful free radicals.

Tannins possess anti-infective, anti-inflammatory, anticarcinogenic, antimutagenic and anti-hemorrhagic properties \& it has been used to treat tonsillitis, pharyngitis, hemorrhoids and skin eruptions \& an antidote for metallic alkaloidal and glycosidic poisons, with which it forms insoluble precipitates [16].

Tannins inhibit the growth of many fungi, yeasts, bacteria, and viruses and it have also been reported to exert physiological effects that are dosedependent, such as to accelerate blood clotting, reduce blood pressure, decrease the serum lipid level, produce liver necrosis and modulate immune-responses [17]. Gallic acid exists as free and as part of hydrolysable tannins. The acid groups are usually polymerized to form dimers such as ellagic acid [18]. Gallic acid possesses antioxidant properties, anticancer, cytotoxicity against cancer cells, without harming healthy cells, anti-parasitic, antimicrobial, anti-inflammatory, bacteriostatic and bactericidal effects, neuroprotective, cardioprotective, gastroprotective, anti HIV effects by inhibiting HIV-1 integrase [19,20]. Gallic acid is used a remote astringent in cases of internal hemorrhage \& to treat albuminuria and diabetes [21]. It is also involved in various signaling pathways that regulate wide range of biological functions including pro- and inflammatory pathways, intrinsic and extrinsic pathways of apoptosis. Gallic acid and its derivatives are safe and stable that can to be used as dietary supplements [19]

Epicatechin (catechin) is a powerful antioxidant. Catechins have a flavon structure and are called flavonoids. Catechins possesses antiviral \& antibacterial activities; useful for treating heart diseases, it may have an effect on endothelium-dependent vasodilation which could improve blood pressure, it reacts with toxins created by harmful bacteria and harmful metals such as lead, mercury, chrome, and cadmium [22,23,24,25,26].

Protocatechuic acid (PCA, dihydroxybenzoic acid) belongs to polyphenols. PCA is a major metabolite of antioxidant polyphenols. It has effects on normal and cancer cells [27]. PCA possesses antioxidant, antibacterial, anticancer, antiulcer, antidiabetic, antiageing, anti-brotic, antiviral, anti-inflammatory, analgesic, cardiac, neurological, nephroprotective, hepatoprotective \& anti-atherosclerotic activities [28, 29]. PCA induces apoptosis of human leukemia cells, as well as malignant HSG1 cells taken from human oral cavities [30]. PCA effectively inhibits the replication of herpes simplex virus type 2 and the activity of urease [31].

Leucocyanidin is a substrate for the enzyme leucocyanidin, 2oxoglutarate: oxygen oxidoreductase (leucoanthocyanidin dioxygenase or anthocyanidin synthase), which participates in flavonoid biosynthesis [32]. Ellagic acid \& epicatechin possess anti-SARS-CoV-2 effects, the two molecules inhibit the activity of the SARS-CoV-2 main protease (Mpro), which has no similarity with the human enzymes [33, 34, 35, 36].

Saponins are glucosides that improve blood cholesterol, bone health and the immunity, they possess anticancer, antitumor and anti-mutagenic activities, they can lower the risk of human cancers, by preventing cancer cells from growing [37, 38].

Phytosterols are plant sterols. They competitively inhibit cholesterol absorption across the gut and thereby can reduce cholesterol levels and this might contribute in reduction the risk of heart diseases [37].

Cyclitols are alcohols multi-hydric cycloalkane, component of cell membrane. They involved in cell regulation, signal transduction, osmoregulation, and ion channel physiology. Cyclitols are efficiently used in the treatment of insulin resistant diabetes, obesity and polycystic ovarian syndrome; they possess anti-atherogenic, anti-oxidative, antiinflammatory and anti-cancer properties [39].

Amines are vital biological compounds essential for human health.

Fibers assist in weight loss, elevate constipation, reduce absorption of fats and cholesterol, absorb many toxins and carcinogenic agents and prevent their absorption, delay the absorption of sugars, help in maintaining the health of the gastro-intestinal tract [40].

Mucilage are polysaccharides, which possess antidiabetes, antioxidant, anticancer, anti-inflammatory, antispasmodic, immuno-modulating \& anti-allergic properties, they improves wound healing, immunity and angiotensin converting enzyme inhibition, they are used as cough suppressants and to treat respiratory disorders \& they are the most proposed materials for modulating drug delivery, mucilage is perfect as natural laxative, and treats a lot of digestive problems, helps cartilage and joint building, maintains joint viscosity \& lowers cholesterol.

\section{[41].}

Proteins, the end products of genes are the basis of life. Cystine, methionine, threonine, lysine and tryptophan in the form of proteins perform multiple of structural, hormonal, antibodies and catalytic functions that are essential for life. Amino acids serve in nerve transmission, regulation of cell growth and the biosynthesis of porphyrins, purines, pyrimidine and urea. Methionine \& tryptophan are nutritionally essential amino acids and the human diet must contain them in quantities adequate to support infant growth to maintain health in adults. Cystine is the major amino acid in the proteins in the hair, skin and horns. Carbohydrates are the major and first sources for energy to the human body. They are precursors for many important cell molecules. They are metabolized to generate reduced nicotinamide adenine dinucleotide phosphate for reductive processes $\&$ other precursors for nucleic acid synthesis (eg. ribose and deoxyribose). They provide metabolites that are used in detoxification reactions \& in the biosynthesis of certain polysaccharides important for normal formation of cartilage, tendons \& bones. Amino-sugars are found in many cell and tissue important structures like blood groups [42].

Gums is used as astringent, emollient, liver tonic, antipyretic and antiasthmatic\& for for stomach upset and pain [7].

Terpenes are essential plant oils. In human, they can induce biosynthesis of neurotransmitters such as serotonin a central nervous system neurotransmitter derived from the amino acid tryptophan involved in regulating mood, sleep, appetite, and sexuality \& dopamine a neurotransmitter involved in motivation, reward, addiction, behavioral reinforcement, and coordination of bodily movement [43].

Lipids are important signaling molecules (such as Eicosanoids and steroid hormones). Fats are storage form of metabolic fuels and are the best heat producers in the body than carbohydrates \& proteins, structural 
components of cell membranes, insulators, thermal \& electrical preventing body from cold, important dietary constituents, due to their high energy content, they are essential for absorption of fat- soluble vitamins. Fats are necessary for sex-maturation, pregnancy and lactation. The significance of dietary fats and oils is that they provide essential fatty acids. Deficiency of EFAs results in impaired brain growth, mental retardation and learning difficulties, dermatitis, hair loss, and poor wound healing. The four fat-soluble vitamins namely vitamin A, D, E and $\mathrm{K}$ are, in fact, require fats and oils in the food to be absorbed through the gut. Inadequate fats may results in the deficiency of these vitamins leading to serious metabolic derangements with subsequent manifestations like night blindness, osteoporosis, bleeding from skin and mucus membranes, phrenoderma, and susceptibility to infections [42]. The phospholipids of cell membranes and of the mitochondrial membranes contain essential fatty acids. Deficiency in essential fatty acids negatively affects the integrity of these membranes and many metabolic processes. Membranes of the red blood cells become fragile and may easily get haemolysed. The skin becomes abnormally permeable to sweat and loss of water from the body may occur. Essential fatty acids are important constituents of lipoproteins. Disposal and oxidation of cholesterol require essential fatty acids \& it is believed that the efficacy of many vegetable oils in diminishing the plasma cholesterol is by virtue of the essential fatty acids content. Arachidonic acid is a direct precursor of prostaglandins in the body. Prostaglandins have several pharmacological effects [42].

Alkaloids are used in the treatment of malaria, asthma, cancer, hypertension, bacterial infections, arrhythmias, diabetes and pain $[44,45,46]$.

Minerals in acacia nilotica fruits such as sodium, potassium, calcium, phosphorus, manganese, magnesium, iron \& copper help promote good health and essential cellular functions.

[46] Conducted antibacterial study to evaluate the efficacy of different extracts of Acacia nilotica fruits against four bacteria species viz Escherichia coli, Staphylococcus aureus, Bacillus subtilus and Pseudomonas aeruginosa. Their results showed the water and the methanolic extracts of the acacia nilotica fruits completely inhibited the growth of the 4 bacteria. Escherichia coli are the cause of $80-85 \%$ of the urinary tract infection in the world which is the second bacterial infections in human population after respiratory tract infections [47, 48]. Currently, antimicrobial resistance the major threat to public health in the World. These make acacia nilotica fruits a very good option to treat UTIs.

\section{Toxicity Studies on Acacia Nilotica Fruits}

There is a misconception about the safety \& toxicity of acacia nilotica fruits. Here, I am giving a brief about the toxicity of acacia nilotica fruits for better understanding its uses.

[49] Investigated the toxicity of acacia nilotica fruit using its aqueous extract on rats in vivo through oral administration for 35 days. Their results revealed no significant disorders occurred in the hematological values, markers of renal and hepatic functions, body weight, absolute and relative organ weights as well as the structures of the kidney and the liver. These results demonstrated the safety of the acacia nilotica fruits in water. [50] Examined the methanol extract of acacia nilotica seeds in vitro to assess its toxicity on lymphocyte. The methanol extracts of seed and husk showed low toxicity effects to the tested lymphocyte at $100 \mathrm{ug} / \mathrm{mL}$. Methanol is known for its toxicity to the brain and is prohibited for human consumption.

[51] Assessed the acute toxicity of acacia nilotica seed aqueous extract on mice in vivo intra-peritoneally \& on cats intravenously, their results demonstrated occasional abdominal cramping.

[52] Investigated the toxicity of acacia nilotica fruit ethanol extract at lower doses on rats in vivo via oral administration for 21 days, their results showed passive behavioral changes of animals during early hours of treatment. But on day 21 all the earlier noted behavioral changes disappeared. Then they used very high doses $50-500 \mathrm{mg} / \mathrm{kg}$ of acacia nilotica fruit ethanol extract intra-peritoneal for 7 and 14 days. The results showed 20-100\% mortality in the tested rats. For safety uses of medicinal herbs, [53] mentioned that most herbal dosages for adults are calculated on the basis of a $150 \mathrm{lb}(70 \mathrm{~kg})$. According to this, if the latter dose of acacia nilotica fruits calculated to adult human it is $35-350 \mathrm{~g} /$ day which are very high doses \& far from the reality. In this context, [54] conducted a research to assess the efficacy of acacia nilotica fruit powder to treat COVID-19, colds, UTIs, tonsillitis \& idiopathic pain, he come to found that the appropriate $\&$ the effective dose for adults was $1.25 \mathrm{~g}$ as a single dose per day after meal for three days and the dose for children was $0.5 \mathrm{~g}$. Also, he found Acacia nilotica fruit powder was very effective in treating teeth carious and pain when it was used topically. His results about the efficacy of the acacia nilotica fruits to treat the mentioned ailments support the existing knowledge and generate new findings.

[55] Studies toxicity of acacia nilotica pods methanol extract on rats for 35 days in vivo via oral route, their results showed no mortality and no toxic reactions.

[56] Studied the toxicity of acacia nilotica pods on goats for 90 days in vivo via oral administration, their result showed the metabolic status of the animal not affected. And when they studied the toxicity of acacia nilotica pods on Caco-2 in vitro, their results showed absence of significant cytotoxicity.

[57] Studied the toxicity of acacia nilotica fruits aqueous extract (100 or $150 \mu \mathrm{g} / \mathrm{mL}$ ) in vivo on rats for 14 days, their results showed no abnormal behavior and no mortality during the treatment.

[58] studied the toxicity of acacia nilotica pods aqueous extract on rats in vivo via oral administration for 21 days, their results demonstrated no death in the treated groups; no significant alteration of the levels of red blood cells, hemoglobin concentration and packed cell volume; while an increase in body weight was observed in day 21 .

[59] studied the toxicity of acacia nilotica pods in Nubian goats fed with whole acacia nilotica pods for 35 days at dose $5 \mathrm{~g} / \mathrm{kg} / \mathrm{d}$, their results showed clinical signs such as salivation, staggered gait, intermittent loss of voice and low appetite; presence of hepatic centrolobular necrosis lesions and fatty changes associated with the significant changes in $\gamma$ glutamyl transferase \& alkaline phosphatase that indicate hepatic dysfunction; renal malfunction is indicated by hemorrhages in addition to the change in urea concentration. Obviously, these adverse effects of acacia nilotica pods were due the extremely high dose and the long period of use. $5 \mathrm{~g} / \mathrm{kg} /$ day of acacia nilotica pods for adult human of $70 \mathrm{~kg}$ weigh are equivalent to $350 \mathrm{~g} / \mathrm{day}$. Our experience demonstrated the effective dose of acacia nilotica fruits for adult human within the range of $1 \mathrm{~g}$ for 3days, maximum for 5 days.

[60] studied the toxicity of acacia nilotica seeds methanol extract on mice in vivo via oral route at doses of $50,100,200,500 \& 1000 \mathrm{mg} / \mathrm{kg}$ body weight, their results showed no mortality in mice during the treatment; little behavioral changes, locomotor ataxia, diarrhea and weight loss were observed. From my experience, the dose $1 \mathrm{~g} / \mathrm{kg}$ of acacia nilotica seeds is extremely high dose; it is equivalent to $70 \mathrm{~g}$ of adult dose of acacia nilotica seeds, although the adverse effects were mild \& might due to methanol, which is known for its toxicity to the brain.

[61] Studied the toxicity of acacia nilotica pods aqueous extract on Rats for 14 days in vivo via oral route; their results showed no mortality at a limit dose of $3000 \mathrm{mg} / \mathrm{kg}$ body weight. Although, the dose is extremely high no mortality occurred. This demonstrates acacia nilotica pods are safe for human use.

[62] Studied the toxicity of acacia nilotica pods aqueous extract in rats maintained on $2 \%$ and $8 \%$ acacia diet for 2 and 4 weeks. Their results showed significant reduction in body weight; no significant changes in serum parameters of hepatic and renal functions, fasting glucose and triglycerides; no mortality and no significant histopathological changes in liver sections were noted. 


\section{Conclusion}

In conclusion, acacia nilotica fruits are very beneficial for human health, safe \& effective to treat various human diseases if it is used in appropriate dose \& period. It has been in use in Sudan \& in other parts of Africa widely \& safely for generations to treat various diseases without any reports of toxicity or adverse effects. Moreover, human body has efficient detoxification mechanisms to process and detoxify toxic substances and toxicity in human occurs only from known high toxic substances, overdose, regular and long period of using of herbs or medicines with known side effects. It is apparent that toxicity of acacia nilotica fruits comes from over dosage \& longer than required duration of uses or regular consumption and the solvent/s as well. However, further works are needed by pharmaceutical industries to authentically formulate various components of the of acacia nilotica fruits to be used as medicines $\&$ supplements to enhance the therapy of several human diseases.

\section{Acknowledgements}

The author is very thankful to his colleagues at the Faculty of Medicine $\&$ Health Sciences, Faculty of Community Development, Faculty of Computer Science \& Information Technology, Faculty of the Sharia \& Law and Faculty of Education, University of Gadarif for their interests in this study and valuable comments. The thankfulness is extended to Prof. Mohamed Zein Ali, the Vice-Chancellor of the University of Gadarif for his support and encouragement.

\section{Conflict of interests}

None.

\section{Funding}

None.

\section{References}

1. Boukandou Mounanga M, Mewono L, Aboughe Angone S. (2015) Toxicity studies of medicinal plants used in sub-Saharan Africa. J. Ethnopharmacol.; 174:618-627.

2. Gurib-Fakim A. (2006) Medicinal plants: traditions of yesterday and drugs of tomorrow. Mol Aspects Med.; 27(1):193.

3. Diallo D, Paulsen BS, Liljeback TH, Michaelsen TE. (2003) the Malian medicinal plant Trichilia emetica; studies on polysaccharides with complement fixing ability. J. Ethnopharmacol.; 84(2-3):279-287.

4. United States Department of Agriculture, Vachellia nilotica. Germplasm Resources Information Network, Agricultural Research Service.

5. Atif Ali, Naveed Akhtar, Barkat Ali Khan, Muhammad Shoaib Khan, Akhtar Rasul, Shahiq-UZ-Zaman, Nayab Khalid, Khalid Waseem, Tariq Mahmood and Liaqat Ali (2012) Acacia nilotica: A plant of multipurpose medicinal uses.

6. Quattrocchi, Umberto (2000). CRC World Dictionary of Plant Names. 1 A-C. CRC Press.

7. Rana D., 2018. A Review of Ethnomedicine, phytochemical and pharmacological Properties of acacia Nilotica (Babool/Kikkar), International J. of Biology, Pharmacy \& Allied Sciences, May, 2018, 7(5): 856-863.

8. Manzo LM, Moussa I, Ikhiri K., 2017. Phytochemical screening of selected medicinal plants used against diarrhea in Niger, West Africa. Int J Herb Med.; 5(4):32-8.9.

9. Mohamed S. Abdalla, Izeldin A. Babiker, Jehan S. Al-Abrahim, Afrah E. Mohammed, Mudawi M. Elobeid, Kamal F. Elkhalifa (2014). Fodder Potential and Chemical Composition of Acacia Nilotica Fruits for Livestock in the Dry lands of Sudan,
International Journal of Plant, Animal \& Environmental Sciences, volume 4. Issue 1, 366-69.

10. Sapna Malviya, Rawat S, Kharia A, Verma M, (2011). Medicinal attributes of Acacia nilotica Linn. A comprehensive review on ethno pharmacological claims. Int. Journal of Pharmacy and Life Science; 2(6):830-837.

11. Seigler DS, (2003) Phytochemistry of Acacia-sensulato. Biochemical Systematics and Ecology. 31(8):845-873.

12. Siddhuraju P, Vijayakumari K, Janardhanan K, (1996) Chemical composition and nutritional evaluation of an under exploited legume, Acacia nilotica (L.) Del. Pharma nutrition, 57(3): 385-391.

13. Lawaly Maman Manzo, Idrissa Moussa, Khalid Ikhiri, Liu Yu (2019). Toxicity studies of Acacia nilotica (L.): A review of the published scientific literature. J Herb med Pharmacol. 2019; 8(3): x-x.

14. Ferrell, Katie E.; Thorington, Richard W. (2006). Squirrels: the animal answer guide. Baltimore: Johns Hopkins University Press.

15. Andrew Pengelly (2004) the Constituents of Medicinal Plants (2nd ed.), Allen \& Unwin, pp. 29-30.

16. Chung KT, Wong TY, Wei CI, Huang YW, and Lin Y. (1998) Tannins and human health: a review Crit Rev Food Sci Nutr. 38(6):421-64.

17. Andrew Pengelly (2004) the Constituents of Medicinal Plants (2nd ed.), Allen \& Unwin, pp. 29-30.

18. Niloofar Kahkeshani, Fatemeh Farzaei, Maryam Fotouhi, Seyedeh Shaghayegh Alavi, Roodabeh Bahramsoltani, Rozita Naseri, Saeideh Momtaz, Zahra Abbasabadi, Roja Rahimi, Mohammad Hosein Farzaei and Anupam Bishayee (2019) Pharmacological effects of gallic acid in health and diseases: A mechanistic review, Iran J Basic Med Sci. 2019 Mar; 22(3): 225-237.

19. Choubey S, Varughese L, Kumar V, Beniwal V. (2015) Medicinal importance of gallic acid and its ester derivatives: a patent review. Pharm Pat Anal.; 4:305-315.

20. Khalesi, S; Sun, J; Buys, N; Jamshidi, A; Nikbakht-Nasrabadi, E; Khosravi-Boroujeni, H (2014). Green tea catechins and blood pressure: A systematic review and meta-analysis of randomised controlled trials. European Journal of Nutrition. 53 (6): 1299-311.

21. Ellinger, S; Reusch, A; Stehle, P; Helfrich, H. P. (2012). "Epicatechin ingested via cocoa products reduces blood pressure in humans: A nonlinear regression model with a Bayesian approach". American Journal of Clinical Nutrition. 95 (6): 1365-1377.

22. Schroeter, H; Heiss, C; Balzer, J; Kleinbongard, P; Keen, C. L.; Hollenberg, N. K.; Sies, H; Kwik-Uribe, C; Schmitz, H. H.; Kelm, M (2006) (-)-Epicatechin mediates beneficial effects of flavanol-rich cocoa on vascular function in humans. Proceedings of the National Academy of Sciences. 103 (4): 1024-1029.

23. Hooper, L; Kay, C; Abdelhamid, A; Kroon, P. A.; Cohn, J. S.; Rimm, E. B.; Cassidy, A (2012). Effects of chocolate, cocoa, and flavan-3-ols on cardiovascular health: A systematic review and meta-analysis of randomized trials. American Journal of Clinical Nutrition. 95 (3): 740-51.

24. Lin HH, Chen JH, Huang CC, Wang CJ (2007). Apoptotic effect of 3,4-dihydroxybenzoic acid on human gastric carcinoma cells involving JNK/p38 MAPK signaling activation. Int J Cancer. 120 (11): 2306-2316.

25. Sahil Kakkar and Souravh Bais (2014) A Review on Protocatechuic Acid and Its Pharmacological Potential, ISRN Pharmacology Volume 2014, Article ID 952943, 9 pages. 
26. Liu, CL; Wang, JM; Chu, CY; Cheng, MT; Tseng, TH (2002). In vivo protective effect of protocatechuic acid on tert-butyl hydroperoxide-induced rat hepatotoxicity. Food Chem Toxicol. 40 (5): 635-641.

27. Babich H, Sedletcaia A, Kenigsberg B (November 2002). In vitro cytotoxicity of protocatechuic acid to cultured human cells from oral tissue: involvement in oxidative stress. Pharmacol. Toxicol. 91 (5): 245-253.31.

28. Hassan STS, Švajdlenka E, Berchová-Bímová K. Hibiscus Sabdariffa L. 2017. PCA Molecules and Its Bioactive Constituents Exhibit Antiviral Activity Against HSV-2 and Anti-enzymatic Properties Against Urease by an ESI-MS Based Assay. 22(5):722.32.

29. Ogundiwin E, Peace C, Nicolet C, Rashbrook V, Gradziel T, Bliss F, Parfitt D, Crisosto C (2008). Leucoanthocyanidin dioxygenase gene (PpLDOX): a potential functional marker for cold storage browning in peach". Tree Genetics \& Genomes. 4 (3): 543-554.

30. Anand Kumar Pandey \& Shalja Verma (5 August 2020) an insilico evaluation of dietary components for structural inhibition of SARS-Cov-2 main protease, J. of Biomolecular Structure and Dynamics.

31. Jin Z., Du X., Xu Y., Deng Y., Liu M., Zhao, Y., Zhang, B., Li, X., Zhang,L., Peng, C., Duan, Y., Yu, J., Wang, L., Yang, K., Liu, F., Jiang, R., Yang,X., You, T., Liu, X., Yang, H. (2020a). Structure of Mpro from SARS-CoV-2 and discovery of its inhibitors. Nature, 582(7811), 289-293.

32. Joshi R. S., Jagdale, S. S., Bansode, S. B., Shankar, S. S., Tellis, M. B.,Pandya, V. K., Chugh, A., Giri, A. P., \& Kulkarni, M. J. (2020) Discovery of potential multi-target-directed ligands by targeting host-specific SARS-CoV-2 structurally conserved main protease. Journal of Biomolecular Structure \& Dynamics, $38,1-16$.

33. Zhang, L., Lin, D., Sun, X., Curth, U., Drosten, C., Sauerhering, L., Becker,S., Rox, K., \& Hilgenfeld, R. (2020) Crystal structure of SARS-CoV-2 main protease provides a basis for design of improved a-keto amide inhibitors. Science (New York, N.Y), 368(6489), 409-412.

34. Hostettmann, K.; A. Marston (1995). Saponins. Cambridge: Cambridge University Press.

35. Agnieszka Owczarczyk-Saczonek, Lesław Bernard Lahuta, Magdalena Ligor, Waldemar Placek, Ryszard Józef Górecki and Bogusław Buszewski (2018). The Healing Promoting Properties of Selected Cyclitols, A Review., Nutrients, 10(12), 1891.

36. Kathleen Zelman, MPH, RD, director of nutrition for WebMD.

37. Ahmad Ameri, Ghazaleh Heydarirad, Jamileh Mahdavi Jafari, AliGhobadi, Hossein Rezaeizadeh \& Rasool Choopani (2015). Medicinal plants contain mucilage used in traditional Persian medicine (TPM), Pharmaceutical Biology, 53:4, 615-623.

38. Ramakrishnan S., Prasannan K.G., \& Rajan R., (1983) Textbook of Biochemistry, ISBN: 0-86-131-239-2, Orient Longman limited, India, pp 10-60; 269; 295-296; 500-525.44.

39. Diana Rangaves (Jun. 30, 2020) what are Cannabis Terpenes? Effects, Health Benefits and How They Compare to cannabinoids-CBD.

40. Kittakoop P, Mahidol C, Ruchirawat S (2014). Alkaloids as important scaffolds in therapeutic drugs for the treatments of cancer, tuberculosis, and smoking cessation. Curr Top Med Chem. 14 (2): 239-252.

41. Cushnie TP, Cushnie B, Lamb AJ (2014). Alkaloids: An overview of their antibacterial, antibiotic-enhancing and antivirulence activities. Int J Antimicrob Agents. 44 (5): 377386.'
42. Qiu S, Sun H, Zhang AH, Xu HY, Yan GL, Han Y, Wang XJ (2014). Natural alkaloids: basic aspects, biological roles, and future perspectives. Chin J Nat Med. 12 (6): 401-406.

43. Abdelhamed A. Abdalla, Mujahed I. Mustafa \& Abdelrafie M. Makhawi (2020). Phytochemical screening and antimicrobial activities studies of Acacia nilotica fruit cover, bioRxiv.

44. Salvatore, S; Salvatore, S, Cattoni, E, Siesto, G, Serati, M, Sorice, P, Torella, M (2011). Urinary tract infections in women.European journal of obstetrics, gynecology, and reproductive biology 156 (2): 131-136.

45. Nicolle LE (2008). Uncomplicated urinary tract infection in adults including uncomplicated pyelonephritis. Urol Clin North Am 35 (1): 1-12.

46. Koube J, Dongmo SS, Guiama VD, Doubla A, Vedekoi J, Goulime M, et al. (2017) Safety assessment of the fruit extract of Acacia nilotica Linn. Willd ex Delile using rat models. Int $\mathrm{J}$ Biosci. 10(4):81-92.

47. El-Tahir A, Satti GM, Khalid SA. (1999) Antiplasmodial activity of selected sudanese medicinal plants with emphasis on Acacia nilotica. Phytother Res. 13(6):474-478.

48. Amos S, Akah PA, Odukwe CJ, Gamaniel KS, Wambede C. The pharmacological effects of an aqueous extract from Acacia nilotica seeds. Phytother Res. 1999; 13(8):683-5.35.

49. Mohammed EYG, Abdulhadi NH, Badico EEM, El-Hadiyah TM. (2011) Toxic potential of ethanolic extract of Acacia nilotica (Garad) in rats. Sudan J Med Sci. 6(1):1-5.

50. Steven D. Ehrlich (2011). University of Maryland Medical Center.

51. Abdalla, Kamal Omer \& Mohammed Ahmed A. Ahmed (2020). Potential of Treating Coronavirus Disease 2019 with Acacia Nilotica Fruit Powder, University of Gadarif.

52. Khan TA, Anjaria JK, Dedhia V, Gohel AK. (2015) Bioactivity guided fractionation and anti-inflammatory activity of Acacia nilotica pods. Int J Pharm Pharm Sci. 7(7):380-383.

53. Paswan JK, Kumar K, Kumar S, Chandramoni, Kumar A, Kumar D, et al. (2016) Effect of feeding Acacia nilotica pod meal on hematobiochemical profile and fecal egg count in goats. Vet World. 9(12):1400-1406.

54. Dikti Vildina J, Kalmobe J, Djafsia B, Schmidt TJ, Liebau E, Ndjonka D. Anti-Onchocerca and anti-Caenorhabditis activity of a hydro-alcoholic extract from the fruits of Acacia nilotica and some Proanthocyanidin derivatives. Molecules. 2017; 22(5).

55. Umaru B, Saka S, Mahre MB, Ojo NA, Dogo HM, Onyeyili PA. Acute toxicity and effects of aqueous pod extract of Acacia nilotica on some haematological parameters and body weight in rats. International Journal of Health and Medical Information. 2015;4(3):37-42.

56. Medani AB, Samia MAE, Ahmed EA. Toxicity of Acacia nilotica (Garad) to Nubian goats. Open Access J Toxicol. $1(2): 1-5$.

57. Munira S, Naim Z, Akter S, Majumber R, Kabir H, Alam B. (2013) Evaluation of analgesic, anti-inflammatory and CNS depression activities of the methanolic extract of Acacia nilotica seed. European J Biol Sci. 5(4):123-130.

58. Auwal MS, Saka S, Shuaibu A, Mairiga IA, Sanda KA, Ibrahim A, et al. (2013) Phytochemical properties and hypoglycemic acticity of the aqueous and fractionated portions of Acacia nilotica (Fabaceae) pod extracts on blood glucose level in normoglycemic Wistar albino rats. J Med Sci. 13(2):111-117. 
59. Al-Mustafa ZH, Dafallah AA. (2000) A study on the toxicology of Acacia nilotica. Am J Chin Med. 28(1):123-129. 